\title{
Téoros
}

Revue de recherche en tourisme

\section{Analyse de certaines variables stratégiques pour l'implantation d'un parc thématique au Québec}

\section{Suzanne Chassé et Pierre Rochon}

Volume 12, numéro 3, octobre 1993

Le renouveau des parcs à thèmes

URI : https://id.erudit.org/iderudit/1077931ar

DOI : https://doi.org/10.7202/1077931ar

Aller au sommaire du numéro

Éditeur(s)

Université du Québec à Montréal

ISSN

0712-8657 (imprimé)

1923-2705 (numérique)

Découvrir la revue

Citer cet article

Chassé, S. \& Rochon, P. (1993). Analyse de certaines variables stratégiques pour l'implantation d'un parc thématique au Québec. Téoros, 12(3), 10-14.

https://doi.org/10.7202/1077931ar d'utilisation que vous pouvez consulter en ligne. 


\section{Analyse de certaines variables stratégiques pour l'implantation d'un parc thématique au Québec Suzanne Chassé et Pierre Rochon"}

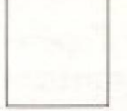

Plusieurs variables stratégiques doivent être analysées en détails préalablement à l'implantation d'un parc thérmatique (localisation et accessibilité, taille, clientèle, calibrage des investissements, rentabilité, impact sur l'environnement, etc.).

Cet article aborde sommairement certaines de ces variables dans une perspective québécoise.

\section{La taille d'un parc thématique}

La taille d'un parc thématique est un facteur qui influence, entre autre, sa localisation et cela de deux façons possibles:

D'une part, la taille d'un parc a une incidence directe sur les espaces requis pour son exploitation, auxquels il est nécessaire de greffer une réserve foncière pour parer aux développements futurs. La disponibilité de ces superficies devient un critère important dans le choix d'une localisation. D'autre part, la taille même d'un parc (qui est fonction de son niveau d'investissement) est un déterminant majeur de sa force d'attraction. Bien que cette relation ne soit pas linéaire, plus la taille d'un parc est grande, plus son pouvoir d'attraction est élevé. Ainsi un pare de plus grande taille permet une plus grande liberté dans le choix de sa localisation. En effet, les clientèlles seront prêtes à faire un effort supplémentaire pour $s^{y} y$ rendre, compte tenu de son pouvoir d'attraction.

À titre d'exemple, le tableau 1 présente la superficie de certains parcs. On peut faire ainsi un lien entre l'aire de rayonnement de l'attractivité du parc et sa superficie (tableau 2).

Madame Suzanne Chasst est protesseure en tourisme au Colltoe de Granby et associbe-expent chez Zins, Beauchesne et Associes. Monsieur Pierre Rochon est 6conomste, M.B. $\mathrm{A}_{\text {if }}$ associd setnior chez Zins; Beauchesne et Associess.

\begin{tabular}{|c|c|c|}
\hline \multicolumn{3}{|c|}{ Tableau 1} \\
\hline Parcs & $\begin{array}{l}\text { Superficie actuelle } \\
\text { du parc } \\
\text { (acres) }\end{array}$ & $\begin{array}{c}\text { Espace tampon } \\
\text { prévu } \\
\text { (acres) }\end{array}$ \\
\hline Canada Wonderland, CDN & 370 & - nd - \\
\hline Zoo de Granby, CDN & 70 & 100 \\
\hline Heritage Park, CDN & 163 & - nd - \\
\hline Six Flags, USA & 100 à 200 & - nd - \\
\hline Darien Lake, USA & 100 & - nd $=$ \\
\hline Bush Garden, USA & 350 & - nd - \\
\hline Astérix, France & 108 & 310 \\
\hline Futuroscope, France & 370,5 & 1235 \\
\hline
\end{tabular}

\section{Tableau 2}

\section{Envergure des parcs}

Parc d'intérêt local

Parc d'intérêt provincial

Parc d'intérêt national

Parc d'intérêt international moins de 200 acres

200 à 250 acres

300 à 400 acres

plus de 400 acres

\section{Tableau 3}

Affectations des espaces du parc Astérix

Parc de loisirs

Extension du parc

Stationnement

Extension stationnement

- Stationnement personnel

- Entretien, aire de service

- Zone de protection

- Zone hôtelière

- Espace boisé

- Voies d'accès

Total
Superficie

(acres)

45,2

24,7

48,7

12,4

6,2

7,4

49,4

29,6

103,7

91,4 
Plus particulièrement, l'exemple du parc Astérix, en banlieue de Paris, nous permet de cerner les besoins en espace pour un parc thématique (tableau 3).

\section{Les prévisions d'achalandage}

Les prévisions d'achalandage constituent une variable stratégique majeure dans la décision de créer un parc thếmatique. Ultimement, la rentabilité financière du projet est directement fonction de cette variable.

Pour la région de Montréal, le marché potentiel de visiteurs dans un rayon de $400 \mathrm{~km}$, plus la clientèle touristique déà présente, s'exprime comme le présente le tableau 4.

Les prévisions d'achalandage peuvent être estimées en appliquant des taux de pénétration à chacun des segments de clientèle potentielle.

Taux de pénétration comparatifs

La firme américaine Economic Research Association (ERA) a établi pour le marché américain des taux de pénétration pour les différents marchés. Ces ratios varient selon deux facteurs : l'éloignement de l'attrait et la valeur des immobilisations.

Les ratios de ERA correspondent au marché américain. Les investisseurs européens qui ont utilisé ces taux lors d'études de faisabilité se sont vite aperçus, après quelques années d'exploitation, qu'ils ne pouvaient appliquer ces ratios dans le contexte européen. L'utilisation de ces ratios a eu comme effet de surévaluer la fréquentation des parcs et les revenus prévisionnels.

Le tableau 5 détermine la fourchette de taux de pénétration, pour chacun des marchés.

- Le marché américain est donc beaucoup plus réceptif que le marché européen, mais la croissance exceptionnelleentreles années 1970 et 1980 de la clientèle des parcs américains, a diminué de beaucoup dans les décennies 1980 et 1990.

Scénarios de fréquentation pour un parc thématique à proximité de Montréal

Pour établir les prévisions d'achalandage d'un parc thématique à

Tableau 4

Clientèle potentielle d'un parc thématique situé dans les environs de Montréal ${ }^{(0)}$

\begin{tabular}{lc}
\hline Zones & Population \\
\hline 0-50 km & 3280000 \\
$51-200 \mathrm{~km}$ & 3200000 \\
. $201-400 \mathrm{~km}$ & 9600000 \\
Marché touristique \\
(tous buts de voyage)
\end{tabular}

Total 21900000

Tableau 5

Ratios comparatifs de taux de pénétration

$\begin{array}{ll}\text { Investissement } & \text { Investissement } \\ \text { inférieur } & \begin{array}{l}\text { supérieur } \\ \text { à } 100 \text { millions } \$^{())}\end{array} \\ \text {(Parc moyen) } 100 \text { millions } \$^{(2)} & \text { (Parc majeur) }\end{array}$

\begin{tabular}{|c|c|c|c|c|}
\hline & Marché & Ratio É-U. & Ratio É.-U. & Ratio Europe $e^{0}$ \\
\hline . & $0-50 \mathrm{~km}$ & 15 à $28 \%$ & 20 à $45 \%$ & $25 \%$ \\
\hline . & $51-200 \mathrm{~km}$ & 5 à $11 \%$ & 10 à $15 \%$ & $2 \%$ \\
\hline . & 201 à $400 \mathrm{~km}$ & 1 à $6 \%$ & $2 \mathrm{a} 35 \%$ & $4 \%$ \\
\hline . & Marché touristique & -..n. & 2 à $35 \%$ & $4 \%$ \\
\hline
\end{tabular}

Tableau 6

Prévisions d'achalandage pour un parc à Montréal ${ }^{(4)}$

(Nombre de visiteurs)

\begin{tabular}{|c|c|c|c|c|}
\hline & \multicolumn{2}{|c|}{ Scénario minimal } & \multicolumn{2}{|c|}{ Scénario maximal } \\
\hline & $\begin{array}{l}\% \text { de } \\
\text { rétention }\end{array}$ & $\begin{array}{l}\text { Nombre } \\
\text { absolu }\end{array}$ & $\begin{array}{l}\% \text { de } \\
\text { rétention }\end{array}$ & $\begin{array}{l}\text { Nombre } \\
\text { absolu }\end{array}$ \\
\hline $0-50 \mathrm{~km}$ & $15 \%$ & 492000 & $20 \%$ & 656000 \\
\hline . $51-200 \mathrm{~km}$ & $6 \%$ & 193000 & $10 \%$ & 322000 \\
\hline $201-400 \mathrm{~km}$ & $1 \%$ & 96000 & $3 \%$ & 288000 \\
\hline - Marché touristique & $1 \%$ & 58000 & $2 \%$ & 116000 \\
\hline Total & & 839000 & & 1382000 \\
\hline
\end{tabular}


Montréal, l'hypothèse des ratios minimaux de l'ERA a été retenue (tableau 6).

En fonction de ces hypothèses, l'achalandageannuel du parc pourrait varier entre 839000 et 1382000 visiteurs. Le scénario réaliste estime à un million de visiteurs, la fréquentation potentielle du parc probable.

\section{Le calibrage des investissements}

La méthodologie retenue pour calibrer les investissements, s'est appuyée sur une analyse de différents sites comparables afin d'établir un ratio de la valeur des immobilisations en fonction de la fréquentation. Ces sites comparables ont été sélectionnés principalement en fonction de leur vocation et de leur niveau de fréquentation.

Rapport entre la valeur des immobilisations et la fréquentation

. Une analyse sommaire de différents sites comparables à travers le monde permet de relever des écarts importants dans les ratios pour les différents pays. Les tableaux ci-dessous illustrent ces différences.

Etats-Unis,

- Aux États-Unis, les attraits touristiquesmajeursontinvestit, en moyenne, 64,80 \$ (dollars canadiens de 1992) pour attirer un visiteur (tableau 7).

France

- Du côté de la France, le ratio est beaucoupplusélevé; ils'établità $168 \$ /$ visiteur. Cettemoyennea étécalculée sur les trois sites présentés au tableau 8.

\section{Canada}

- Au Canada, le même exercice, réalisé à partir d'un échantillon plus diversifié d'attraits ou de projets, permet d'érablir le ratio moyen investissement/visiteur à 113 \$ (tableau 9).

, À partir de ces informations, il apparait clairement que les investissements requis par visiteur demeurent importants (tableau 10).

- Une partie importante des écarts entre ces ratios est sûrement attribuable à des facteurs propres, aux différents pays concernés. Les États-Unis, par exemple, bénéficient d'une densité de population et de touristes nettement plus grande qu'au Canada. Parallèle-
Tableau 7

Ratios immobilisations/fréquentation des parcs américains

\begin{tabular}{|c|c|c|}
\hline Attraits & $\underset{\text { 1983/visiteur }}{\text { S US }}$ & $\begin{array}{c}\text { S CA - } \\
\text { 1992/visiteur(s) }\end{array}$ \\
\hline Magic mountain & $32,7 \mathrm{~s}$ & $58,3 \mathrm{~S}$ \\
\hline Cedar Point & $37,0 \mathrm{~S}$ & $66,0 \$$ \\
\hline King island & $37,0 \mathrm{~S}$ & $66,0 \mathrm{~S}$ \\
\hline Great adventure & $36,4 \mathrm{~S}$ & $64,9 \mathrm{~S}$ \\
\hline Great america & $38,5 \mathrm{~S}$ & $68,7 \mathrm{~S}$ \\
\hline Moyenne & $36,32 \mathrm{~S}$ & $64,8 \mathrm{~S}$ \\
\hline
\end{tabular}

Tableau 8

Ratios d'immobilisations/fréquentation des parcs français

\begin{tabular}{lc}
\hline \multicolumn{1}{c}{ Attraits } & \$CA $-1992 /$ Visiteur \\
\hline Astérix & $136 \$$ \\
Futuroscope & $200 \$$ \\
Wallaby-Schtroumph & $168 \$$ \\
\hline Moyenne & $168 \$$ \\
\hline
\end{tabular}

Tableau 9

Ratio immobilisations/fréquentation des attraits canadiens

Attraits

Immobilisations Fréquentation

ratio (millions \$ CA 1992)(millions de visiteurs) \$/visiteur

\begin{tabular}{lccr}
\hline $\begin{array}{l}\text { Zoo de Granby } \\
\begin{array}{l}\text { Heritage Park } \\
\text { (Calgary) }\end{array}\end{array}$ & 80 &, 5 & $160 \mathrm{~S}$ \\
$\begin{array}{l}\text { Village d'Antan } \\
\text { (Drummondville) }\end{array}$ & 10 &, 435 & $138 \$$ \\
$\begin{array}{l}\text { Musée science et } \\
\text { technologie }\end{array}$ &, 106 & $94 \mathrm{~S}$ \\
$\begin{array}{l}\text { (Projet à Montréal) } \\
\text { Canada Wonderland }\end{array}$ & 100 & 1,1 & $91 \$$ \\
$\begin{array}{l}\text { (Ontario) } \\
\text { La Ronde }\end{array}$ & 250 & 2,2 & $114 \$$ \\
\hline
\end{tabular}

Moyenne

$113 \$$ 


\section{Tableau 10}

Moyenne des ratios d'immobilisations en fonction de la fréquentation
Pays
Ratio
(\$ CA 1992/visiteur)

\section{U.S.A.}

$65 \$$

France

$168 \$$

Canada

$113 \$$

\section{Moyenne}

$115 \$$

\section{Tableau 11}

Évaluation des investissements pour un parc thématique dans la région de Montréal

\begin{tabular}{lccc}
\hline scénarions & $\begin{array}{c}\text { fréquentation } \\
\text { (millions \$CA 1992) }\end{array}$ & \multicolumn{2}{c}{$\begin{array}{c}\text { investissements } \\
\text { (millions de visiteurs) }\end{array}$} \\
\hline & & $\begin{array}{c}\text { minimun } \\
(100 \$ / \text { visiteur) }\end{array}$ & $\begin{array}{c}\text { maximun } \\
(125 \text { \$/visiteur) }\end{array}$ \\
\cline { 3 - 4 } minimal & 0,84 & $84 \$$ & $105 \$$ \\
intermédiaire & 1,0 & $100 \$$ & $125 \$$ \\
maximal & 1,4 & $140 \$$ & $175 \$$ \\
\hline
\end{tabular}

Tableau 12

Sources des revenus d'exploitation

\section{Sources des} revenus

Entrées

Restaurants

Commerces

Divers

\section{$\%$ des}

revenus totaux d'exploitation
50 à $55 \%$

15 à $20 \%$

20 à $25 \%$

5 à $10 \%$ ment, en France, les ratios êlevés sont explicables en partie par la faiblesse de la fréquentation due au peu de notoriété et de crédibilité de ces attraits auprès de la population; ils sont considérés comme des sous-produits culturels et sont souvent boycottés par l'élite et la presse.

Les données canadiennes sont donc sûrement plus représentatives pour unéventuel projet decréation de parcs thématiques au Québec et elles situeraient le ratio d'investissement par visiteur à 113 \$.

Calibrage des investissements pour un parc thématique dans la région de Montréal

Afin de calibrer les investissements, deux variables seront retenues pour le calcul : l'investissement par visiteur et le nombredevisiteurs prévus (tableau 11).

- Tel que discuté précédemment, l'investissement pourl'implantation d'un attrait touristique majeur à Montréal pourrait sechiffrerentre 100 Set $125 \mathrm{~S}$ par visiteur.

Par ailleurs, en se référant aux scénarios de fréquentation établis initialement, les investissements requis pourraient donc être évalués entre 84 millions $\$$ et 175 millions \$.

Si le parc thématique avait une fréquentation moyenne de 1 million de visiteurs par année, un investissement minimum de 100 millions \$ devrait être envisagé.

- Le nombre de visiteurs pourrait être plusélevé, si l'originalité de l'attraitet les efforts marketing consentisétaient plus importants, permettant ainsi d'augmenter le pouvoir d'attractivité sans en augmenter les coûts.

- Pour limiter l'investissement à 100 millions $\$$ tout en maintenant un objectifde fréquentation del'ordre de 1 million de visiteurs, les promoteurs et les partenaires d'un tel parc devraient nécessairement être plus «performants» que la moyenne dans la conception, le développement, l'implantation etla mise en marchédu parc.

\section{La rentabilité des activités d'exploitation}

L'analyse de la rentabilité des activités d'exploitation s'appuie sur les revenus estimés, les coûts prévus et la marge bénéficiaire.

\section{Revenus}

- La structure des revenus d'exploitation d'un parc thématique prend généralement la forme présentée au tableau 12.

- Ces moyennes constituent des données relativement stables d'un site à un autre. Le coût des entrées et les autres revenus sont par contre des données beaucoup plus variables.

- Pour un parc ayant un prix moyen d'entrée par visiteur (adulte, enfant, âge d'or, groupes, etc.) de l'ordre de $15 \mathrm{\$}$ et un scénario de fréquentation de un million de visiteurs, les revenus découlant uniquement de l'exploita- 
tion pourraient se chiffrer approximativement à 30 millions $\$$.

À ces revenus on pourrait ajouter :

les commandites;

les dons privés;

les subventions de fonctionnement;

etc.

Ces diverses sources de revenus sont toutefois très variables en fonction des pays, des sites, de la structure de propriété, des activités commerciales périphériques, etc.

- Aux États-Unis, par exemple, les commandites constituent une source de revenus généralement très importante et représentent, dans certains cas, entre $10 \%$ et $20 \%$ des revenus totaux des attraits. Par contre, en Europe, les commandites pour les *attraitss touristiques ne sont pas très fréquentes. Demême, au Canada, elles représentent généralement, une proportion beaucoup moins importante des revenus. Cette constatation pour le Canada est intimement liée à la structure de propriété, ou du moins à l'origine des fonds qui servent au financement des infrastructures.

Ainsi, au Canada, la grande majorité des attraits touristiques majeurs sont financés directementou indirectement par l'Etat. Ils sont souvent gérés par des organismes eparapublics ou *paramunicipauxw. Lesautresattraits sont administrés par des organismes sans but lucratif (musées, zoo, etc.).

\section{Coûts}

Toutcomme pour les investissements, une analyse de certaines données financières relatives à des attraits comparables a été réalisée pour établir un ratio coûts d'exploitation/visiteur. Pour les fins de l'étude, les coûts d'exploitation incluent tous les coûts d'opération incluant les coûts directs des commerces générateurs de revenus, les frais d'administration et de marketing mais excluant les frais financiers et les remboursements de capital.

Sauf certaines exceptions, AstérixFrance ( $35 \$ /$ visiteur) et WallabyBelgique (18\$/visiteur), les ratios de dépenses par visiteur se situent généralement entre $20 \mathrm{~S}$ et $30 \mathrm{\$}$; la moyenne est de $26 \mathrm{~S}$ par visiteur.

Pour le Canada, la moyenne apparaît légèrement supérieure et se situe aux environs de $28 \$$ /visiteur. Ce ratio correspond exactement à la situation duJardin Botanique de Montréal pour l'exercice financier de 1992.

- Les coûts d'exploitation les plus importants sont les salaires ( $40 \%$ à 50 $\%)$, la publicité $(10 \%$ à $15 \%)$, les taxes $(10 \%)$. Il faut aussi tenir compte delanécessitédecréer une «provision» pour le renouvellement des équipements et attraits qui n'est pas incluse dans ces coûts d'exploitation.

\section{Marge bénéficiaire}

La marge bénéficiaire avant financement représente les liquidités annuellement disponibles pour assumer les coûts du service de la dette.

. Aux États-Unis, où la majorité des attraits sont privés, les *disponibilités» financières annuelles permettent de financer une portion importante des immobilisations. Il faut toutefois rappeler que plusieurs facteurs «favorisento ces investisseurs :

le ratio investissement/visiteur est beaucoup moins élevé (la forte densité de touristes et de population permet une fréquentation plus élevée);

les attraits ont été implantés par phase sur une longue période de temps;

- le marketing est agressif;

- les commandites sont une source très importante de revenus;

- plusieurs coûts d'exploitation (énergie, etc.) sont moins élevés.

En Europe, la situation est fort différente; il en coûte presque trois fois plus cher par visiteur pour implanter un attrait. Ainsi, en France par exemple, une forte proportion des sites touristiques majeurs sont largement financés par l'Etat (département, région, national) et à l'occasion totalement financés par l'un des paliers gouvernementaux (touriscope).

- Les attraits touristiques majeurs implantés récemment en France et qui ont eu à supporter un financement bancaire important, n'arrivent tout simplement pas à rembourser ces emprunts. Le parc Astérix est l'exemple d'un site qui n'arrive pas à remplir ses engagements. Le financement bancaire est trop élevé car les prévisions de fréquentation n'ont pu être atteintes et conséquemment, la marge bénéficiaire dégagée n'est pas suffisante pour rembourser leurs emprunts.
Pourtant les promoteurs avaient investi l'équivalent de $40 \%$ des immobilisations.

- Au Canada, la situation s'apparente beaucoupplus à celledela Francequ"à celle des Etats-Unis. Les attraits touristiques majeurs ( 1 million de visiteurs et plus) sont soit publics ou financés très majoritairement par des fonds publics. Les marges bénéficiaires ne permettent pas de financer des emprunts bancaires importants.

\section{Le financement}

En se basant sur les analyses préoédentes et en excluant les revenus *extraordinaires», un parc thématique au Québec devrait viser une structure de financement comportant un maximum de $15 \%$ à $25 \%$ d'investissement sous forme de dette remboursable pour assurer sa viabilité.

Ce scénario suppose une performance globale du site supérieure à la moyenne canadienne. Les liquidités générées annuellement par les opérations ne pourront servir exclusivement à financer les immobilisations «originalesm. Unepartiedecesargents devrait nécessairementêture réinvestie pour renouveler les attraits au fil des ans afin d'assurer la viabilité à long terme du site (soit $10 \%$ à $15 \%$ des immobilisations de départ tous les deux ans).

Le financement d'un parc thérmatique au Québec peut donc difficilement se réaliser sans l'appui :

de partenaires majeurs qui pourraient avoirl'opportunitéderentabiliser leur investissement non pas par les opérations du parc mais plutôt par les retombées «économiques» directes et/ ou indirectes;

des différents paliers gouvernementaux (municipal, provincial, fédéral).

\section{Notes}

(1) Données de 1991.

(2) Róactualisation des investissements en dollars canaciens de 1992 des ratios de lafirme Economic Research Association.

(3) Laurence Corriger, * Lesparcs à thèmes, Dossler Espace, no 9, p. 31

(4) Donnees de 1991.

(5) Taux moyen d'inflation $5 \%$ - taux de change $15 \%$. 